\title{
Development and Mechanism of High-Performance Fully Biobased Shape Memory Benzoxazine Resins with a Green Strategy
}

Xin-Long Sha, Li Yuan, Guozheng Liang,* Aijuan Gu*

State and Local Joint Engineering Laboratory for Novel Functional Polymeric Materials

College of Chemistry, Chemical Engineering and Materials Science

Soochow University, 199 Ren’Ai Road, Suzhou 215123, China

- 20 pages

- 4 figures

- 1 table

* Corresponding author. Tel: +86 512 65880967. Fax: +86 512 65880089. E-mail address: lgzheng@suda.edu.cn (Guozheng Liang), or, ajgu@suda.edu.cn (Aijuan Gu). 


\section{Table of contents}

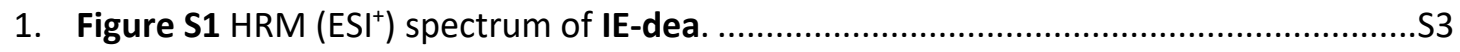

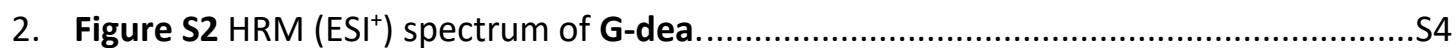

3. Figure S3 Angle measurement snapshots of poly(IE-dea). ..........................................S5

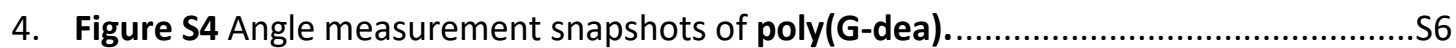

5. Table S1 Key properties of biobased SMPs in this work and literatures..........................S7 


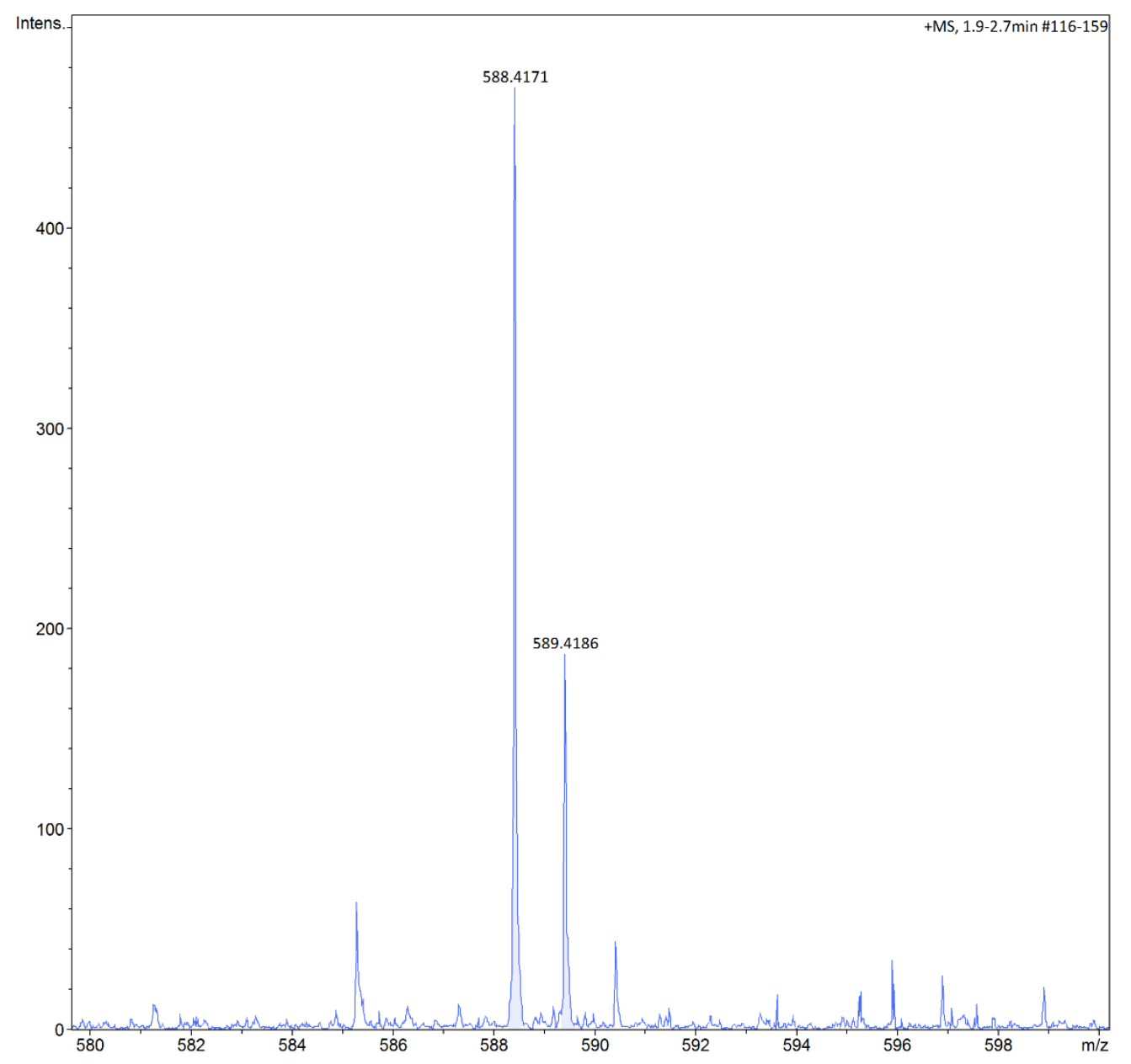

Figure S1 HRM $\left(\mathrm{ESI}^{+}\right)$spectrum of IE-dea. 


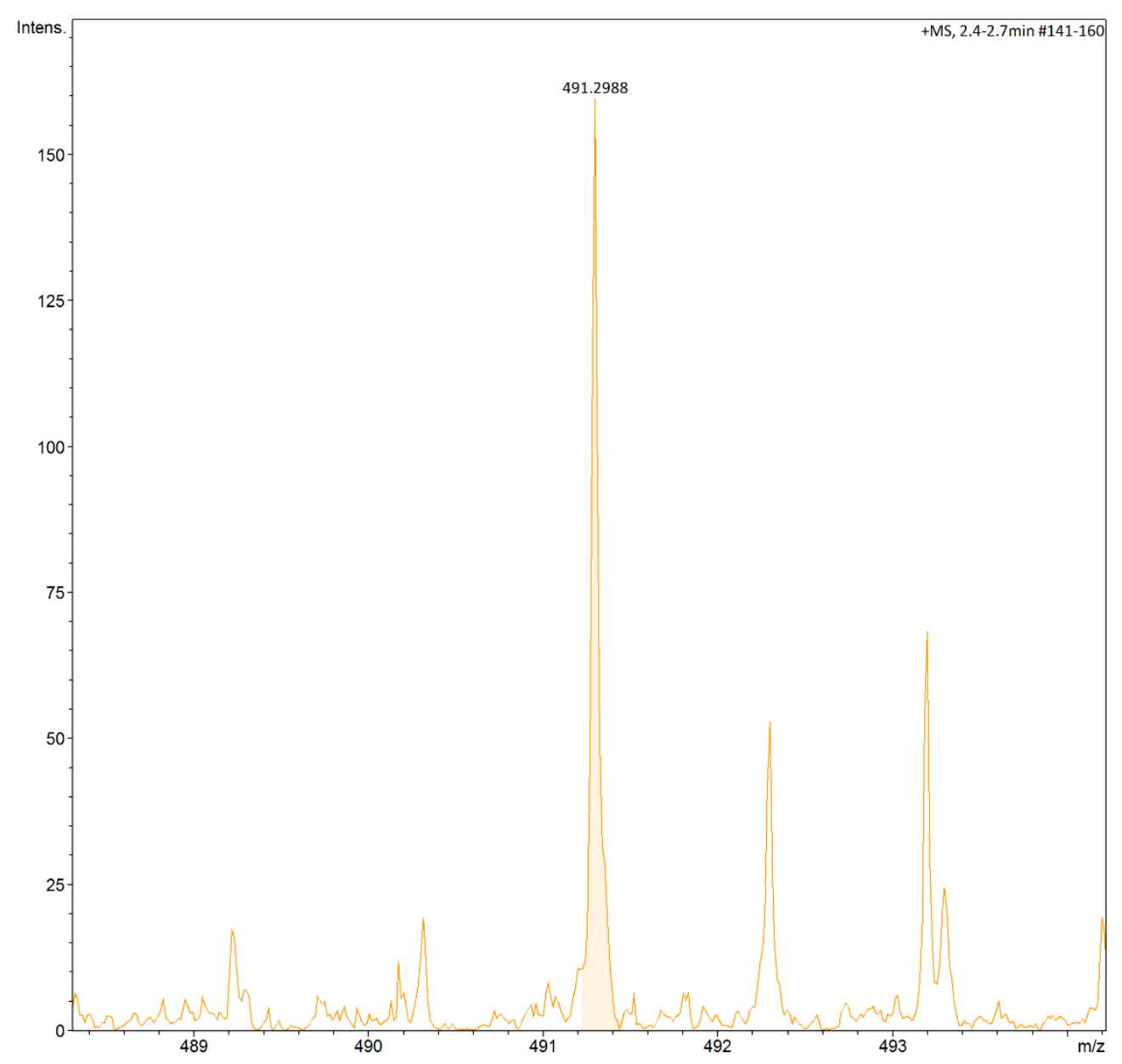

Figure S2 HRM (ESI ${ }^{+}$) spectrum of G-dea. 


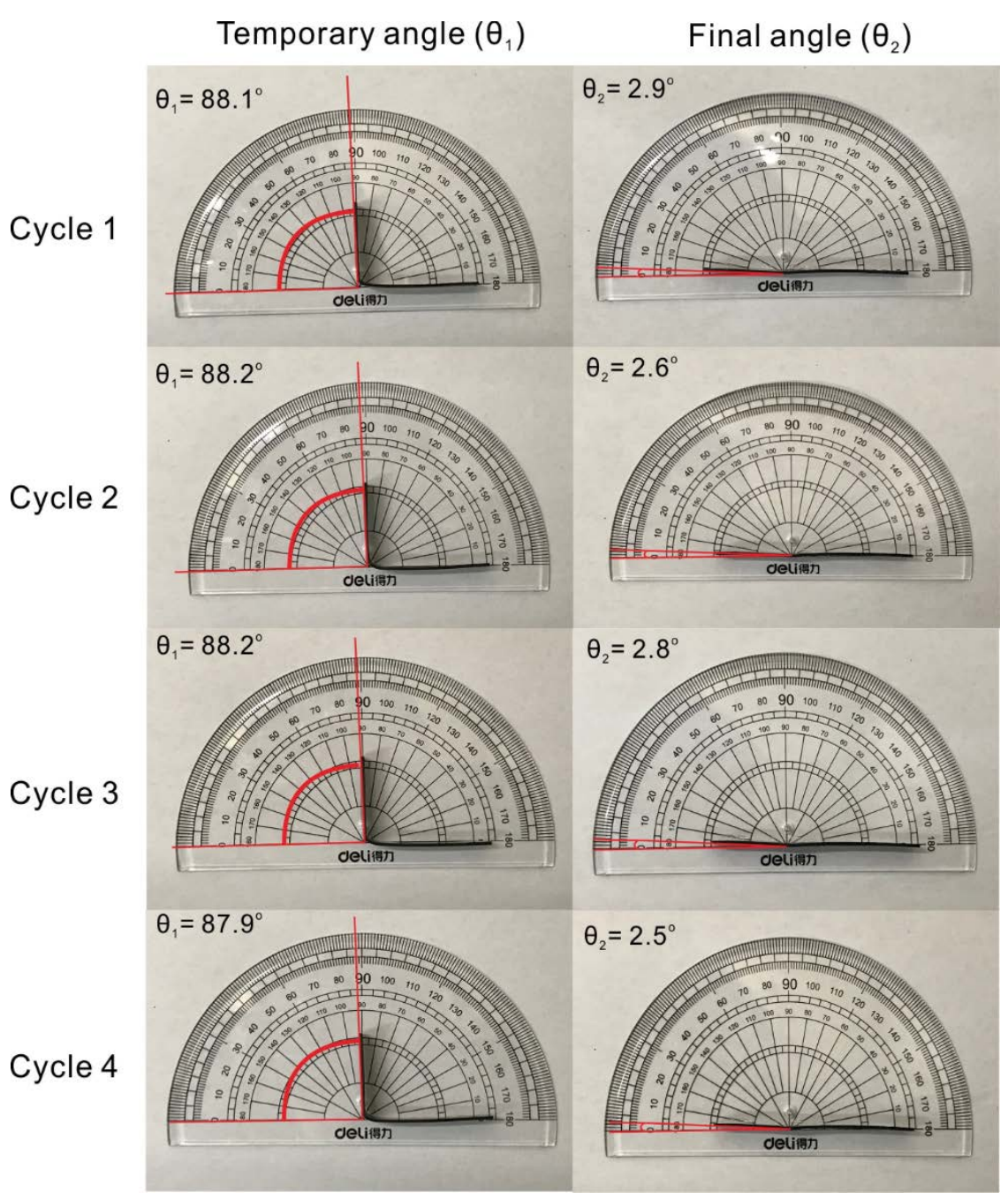

Figure S3 Angle measurement snapshots of poly(IE-dea). 


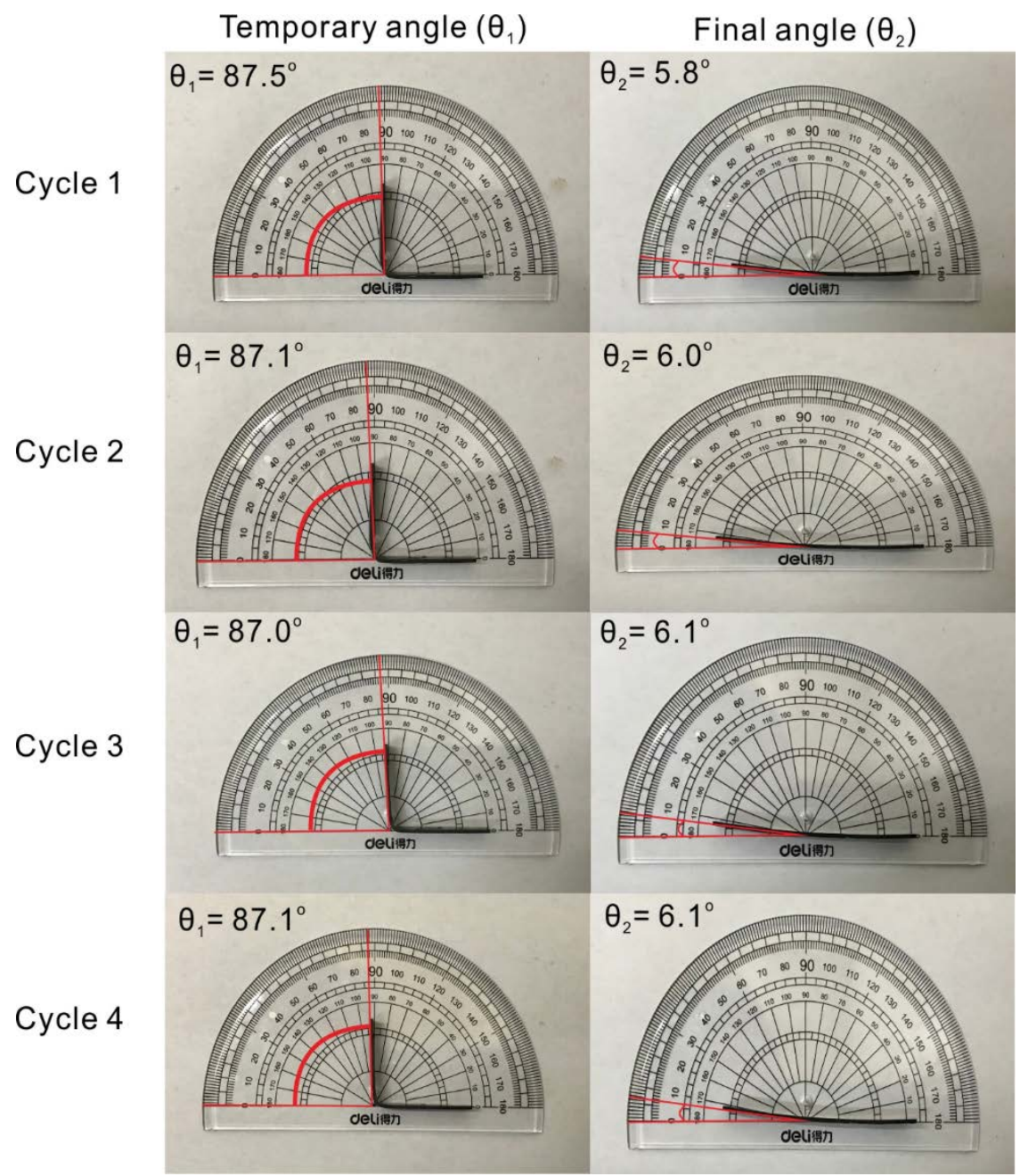

Figure S4 Angle measurement snapshots of poly(G-dea). 
Table S1 Key properties of biobased SMPs in this work and literatures.

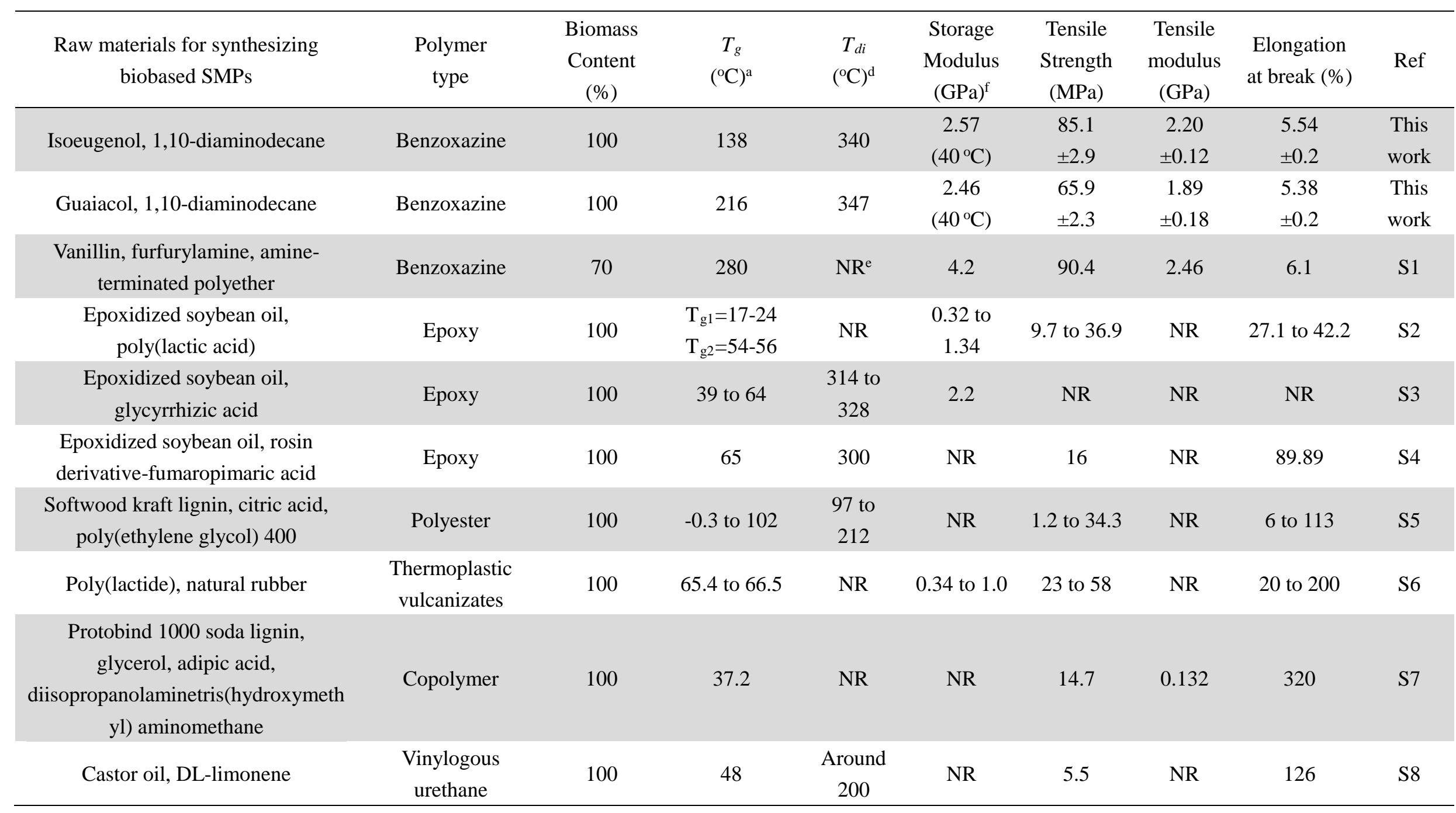




\begin{tabular}{|c|c|c|c|c|c|c|c|c|c|}
\hline $\begin{array}{c}\text { Tannic acid, diglycidyl ether of } \\
\text { bisphenol A }\end{array}$ & Epoxy & 17 & 95.2 to $113.8^{\mathrm{b}}$ & NR & $\begin{array}{l}1.94 \text { to } \\
2.87\end{array}$ & $\begin{array}{c}75.4 \text { to } \\
98.4\end{array}$ & NR & 4.2 to 6.4 & S9 \\
\hline $\begin{array}{l}\text { Palm oil, 2-(methylamino) ethanol, } \\
\text { citric acid monohydrate }\end{array}$ & Epoxy & 65.2 & 35.1 to $65.2^{\mathrm{c}}$ & $\begin{array}{l}\text { Around } \\
250 \text { to } \\
305\end{array}$ & NR & 1.2 to 28.9 & NR & 12 to 109 & $\mathrm{~S} 10$ \\
\hline $\begin{array}{l}\text { Gallic acid, 3-allyl bromide } \\
\text { diglycidyl ether of } \\
\text { bisphenol A }\end{array}$ & Epoxy & 35.4 & 30.4 & 301 & NR & 26.4 & NR & 203 & S11 \\
\hline $\begin{array}{l}\text { Epoxy soybean oil, vanillin } \\
\text { diethylenetriamine }\end{array}$ & Epoxy & 72 & 75.7 & NR & 1.582 & 20 & NR & 12 & $\mathrm{~S} 13$ \\
\hline $\begin{array}{l}\text { Rosin acid, acrylic acid, 2- } \\
\text { aminopropyl ether }\end{array}$ & Epoxy & 51.7 & 110 & 317 & NR & 62 & 0.779 & 13.2 & S14 \\
\hline $\begin{array}{l}\text { Eugenol, zinc acetylacetonate } \\
\text { hydrate, 1,4-dibromobutane, succinic } \\
\text { anhydride }\end{array}$ & Epoxy & 64.3 & 58 & 310 & NR & 25 & NR & 8 & S15 \\
\hline $\begin{array}{l}\text { Vanillin, furfurylamine, epoxidized } \\
\text { castor oil }\end{array}$ & $\begin{array}{c}\text { Benzoxazine/epox } \\
\text { y copolymer }\end{array}$ & 99 & 14 to 144 & NR & NR & 3 to 23 & NR & 1.2 to 5.9 & S17 \\
\hline
\end{tabular}




\begin{tabular}{|c|c|c|c|c|c|c|c|c|c|}
\hline $\begin{array}{l}\text { Bisphenol A diglycidyl ether, cardol, } \\
\text { cardanol, anacardic acid }\end{array}$ & $\begin{array}{l}\text { Epoxy/ } \\
\text { polycaprolactone } \\
\text { copolymer }\end{array}$ & 41 & $35^{c}$ & NR & NR & 12.52 & NR & $\begin{array}{c}\text { Around } \\
32.5\end{array}$ & S18 \\
\hline $\begin{array}{l}\text { Polylactide, epoxidized natural } \\
\text { rubber, } \mathrm{Fe}_{3} \mathrm{O}_{4}\end{array}$ & $\begin{array}{l}\text { Thermoplastic } \\
\text { vulcanizates }\end{array}$ & 76.9 & 62 & NR & NR & 22.62 & 0.763 & 62.72 & S20 \\
\hline $\begin{array}{l}\text { Eucommia ulmoides gum, } \\
\text { polyolefin elastomer }\end{array}$ & $\begin{array}{l}\text { Thermoplastic } \\
\text { vulcanizates }\end{array}$ & 70 & -30 & NR & NR & 18.7 & NR & 1150 & S21 \\
\hline $\begin{array}{l}\text { Poly(lactide), ethylene-glycidyl } \\
\text { methacrylate-vinyl acetate }\end{array}$ & $\begin{array}{l}\text { Thermoplastic } \\
\text { vulcanizates }\end{array}$ & 30 & Around 58 & NR & $\begin{array}{c}0.045 \text { to } \\
0.2\end{array}$ & 2.2 to 12.3 & NR & 310 to 4200 & $\mathrm{~S} 22$ \\
\hline $\begin{array}{c}\text { Polylactide, epoxidized natural } \\
\text { rubber }\end{array}$ & Vulcanizates & 98.5 & 61.7 to 63.8 & NR & $\begin{array}{c}0.151 \text { to } \\
0.657\end{array}$ & $7-14$ & NR & 100 to 250 & $\mathrm{~S} 25$ \\
\hline $\begin{array}{l}\text { Tris(2-aminoethyl)amine, } \\
\text { tris(2-(methylamino)ethyl)amine, } \\
\text { dimethylcarbonate }\end{array}$ & $\begin{array}{l}\text { Polyhydroxyuretha } \\
\text { nes }\end{array}$ & 52 & 20 to 42 & $\begin{array}{c}214 \text { to } \\
219\end{array}$ & $\begin{array}{c}0.00027 \text { to } \\
0.002\end{array}$ & NR & NR & NR & S26 \\
\hline $\begin{array}{l}\text { Diphenylmethane diisocyanate-50, } \\
\text { polylactide diol, polycaprolactone }\end{array}$ & Polyurethane & 90 & 32.5 & 273.1 & NR & 12.9 & 0.00153 & 1345 & S27 \\
\hline
\end{tabular}




\begin{tabular}{|c|c|c|c|c|c|c|c|c|c|}
\hline diol & & & & & & & & & \\
\hline $\begin{array}{l}\text { Hexamethylene diisocyanate, 4,4'- } \\
\text { diphenyl methane diisocyanate, 1,4- } \\
\text { butanediol, }\end{array}$ & Polyurethane & 87.3 & $42.7^{b}$ & 270.8 & NR & 23.5 & 0.38 & 580 & S28 \\
\hline $\begin{array}{c}\text { Poly( } \varepsilon \text {-caprolactone)diol, } \\
\text { monoglyceride, 1,4-butanediol }\end{array}$ & Polyurethane & 90 & NR & $\begin{array}{l}\text { Around } \\
250\end{array}$ & NR & $\begin{array}{c}11.48 \text { to } \\
19.31\end{array}$ & NR & 497 to 835 & S29 \\
\hline 1,4-Pentanediol, succinic acid & Polyurethane & 91.3 & -52 & NR & NR & NR & NR & 136 to 936 & S30 \\
\hline $\begin{array}{c}\text { Polyether-based thermoplastic } \\
\text { polyurethane, freezedried chitosan } \\
\text { fiber }\end{array}$ & Polyurethane & 5 & $52.2^{b}$ & NR & NR & NR & NR & NR & S31 \\
\hline $\begin{array}{l}\text { Castor oil-based macrodiol, } \\
\text { L-LDI, 1,3-propanediol } \\
\text { chitin nanocrystals }\end{array}$ & Polyurethane & 75 & 20.2 & NR & NR & 6.8 to 8.9 & NR & 23 to 870 & S32 \\
\hline $\begin{array}{l}\text { M. ferrea L. seeds, sunflower oil, } \\
\text { castor oil, glycerolpoly(e- } \\
\text { caprolactone) diol }\end{array}$ & Polyurethane & Around 20 & NR & 243 & NR & 7.5 & NR & 620 & S33 \\
\hline Castor oil, polycaprolactone diol & Polyurethane & 3.4 & Around -15 & 220 & NR & 4.6 to 12.8 & NR & $\begin{array}{c}1027.3 \text { to } \\
1233.1\end{array}$ & S34 \\
\hline $\begin{array}{l}\text { 4,4'-Diphenylmethane diisocyanate, } \\
\text { hexamethylene diisocyanate, } \\
\text { dicyclohexylmethane-4,4'- } \\
\text { diisocyanate, castor oil }\end{array}$ & Polyurethane & 73.5 & 40 & $\begin{array}{c}223 \\
\left(\mathrm{~T}_{\mathrm{d} 10}\right)\end{array}$ & NR & 6.87 & NR & 195 to 388 & S35 \\
\hline $\begin{array}{l}\text { Methyl ester of hydroxynonanoic } \\
\text { acid, 1,4-butanediol }\end{array}$ & Polyurethane & $<50$ & 6.64 to 14.41 & NR & NR & $\begin{array}{c}10.0 \text { to } \\
18.4\end{array}$ & NR & 70 to 487 & S36 \\
\hline $\begin{array}{l}\text { Adipic acid, itaconic acid, 1,10- } \\
\text { decanediol, 1,4-butanediol, }\end{array}$ & Polyurethane & 70 & -3.5 to 60 & NR & NR & $\begin{array}{l}13.2 \text { to } \\
40.62\end{array}$ & $\begin{array}{c}0.0038 \\
\text { to } 0.321\end{array}$ & 380 to 1120 & S37 \\
\hline
\end{tabular}




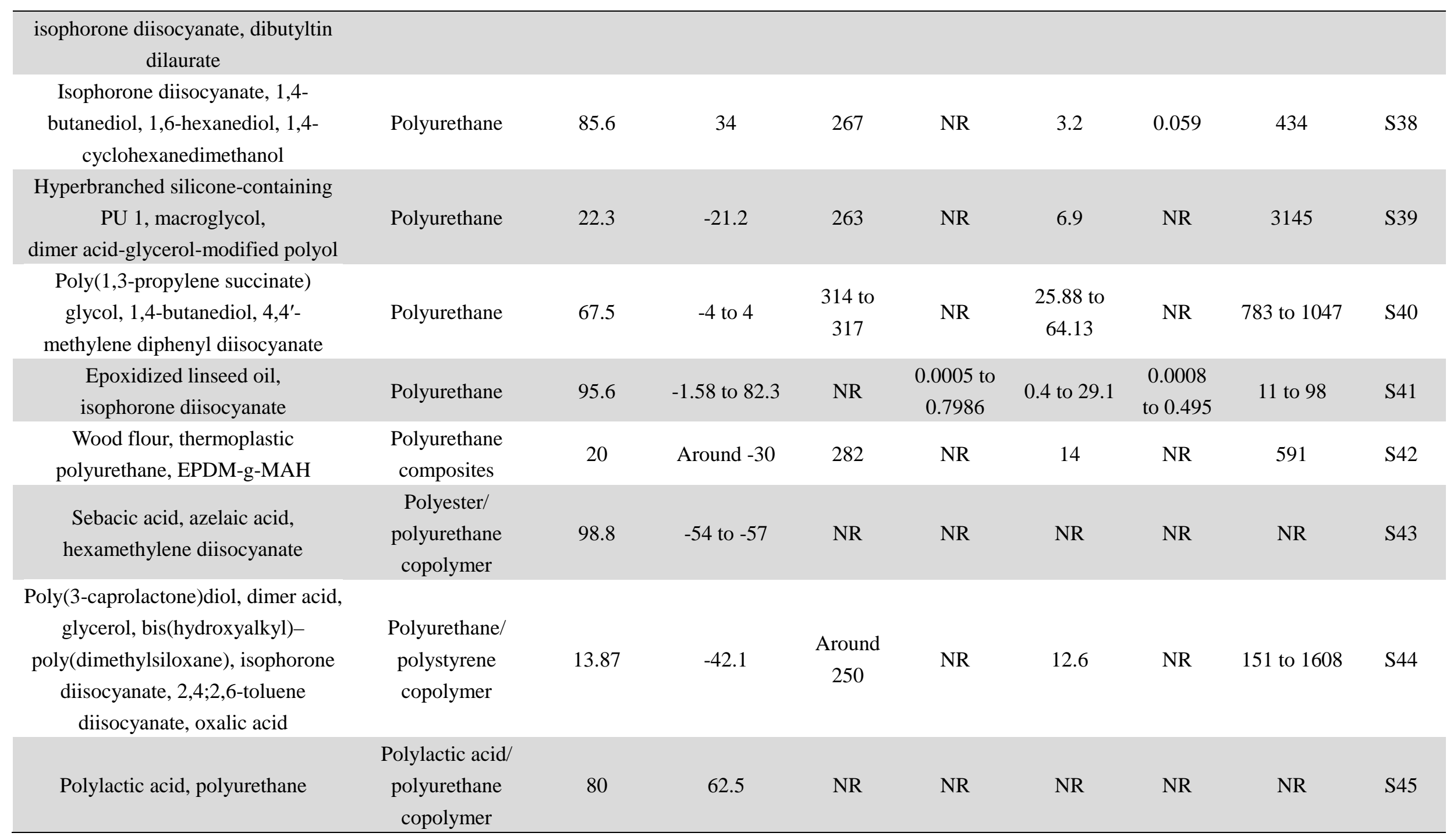




\begin{tabular}{|c|c|c|c|c|c|c|c|c|c|}
\hline $\begin{array}{c}\text { Polyurethane acrylates, } \\
\text { polycaprolactone } \\
\text { diolshexamethylene diisocyanate, } \\
\text { hydroxy-methyl methacrylate }\end{array}$ & $\begin{array}{l}\text { Polyurethane } \\
\text { acrylate }\end{array}$ & 91.5 & -58.1 & NR & NR & 24.4 & 0.1752 & 824.2 & S46 \\
\hline $\begin{array}{c}\text { Itaconic acid, sebacic acid, succinic } \\
\text { acid, 1,3-propane diol }\end{array}$ & Copolyester & 99 & Around 60 & NR & NR & $\begin{array}{l}\text { Around } \\
0.5 \text { to } 4\end{array}$ & NR & 80 to 250 & S49 \\
\hline $\begin{array}{c}\text { Itaconic acid, sebacic acid } \\
\text { 1,4-butanediol, 1,4;3,6-dianhydro-D- } \\
\text { glucitol }\end{array}$ & Copolyesters & 99 & $-34.8^{\mathrm{b}}$ & 370 & NR & 6.1 to 16.4 & NR & 240 to 430 & S50 \\
\hline Amorphous PU, poly(caprolactone) & $\begin{array}{l}\text { Polyester/ } \\
\text { graphene } \\
\text { nanoplatelets } \\
\text { composites }\end{array}$ & 50 & -41.3 to -27.4 & NR & NR & $\begin{array}{c}\text { Around } 8 \\
\text { to } 10\end{array}$ & NR & $\begin{array}{c}\text { Around } 25 \\
\text { to } 350\end{array}$ & S53 \\
\hline $\begin{array}{l}\text { Softwood kraft lignin, } \\
\text { pentaerythritol }\end{array}$ & Poly(caprolactone) & 30 & $-57^{b}$ & NR & NR & NR & NR & NR & S54 \\
\hline
\end{tabular}




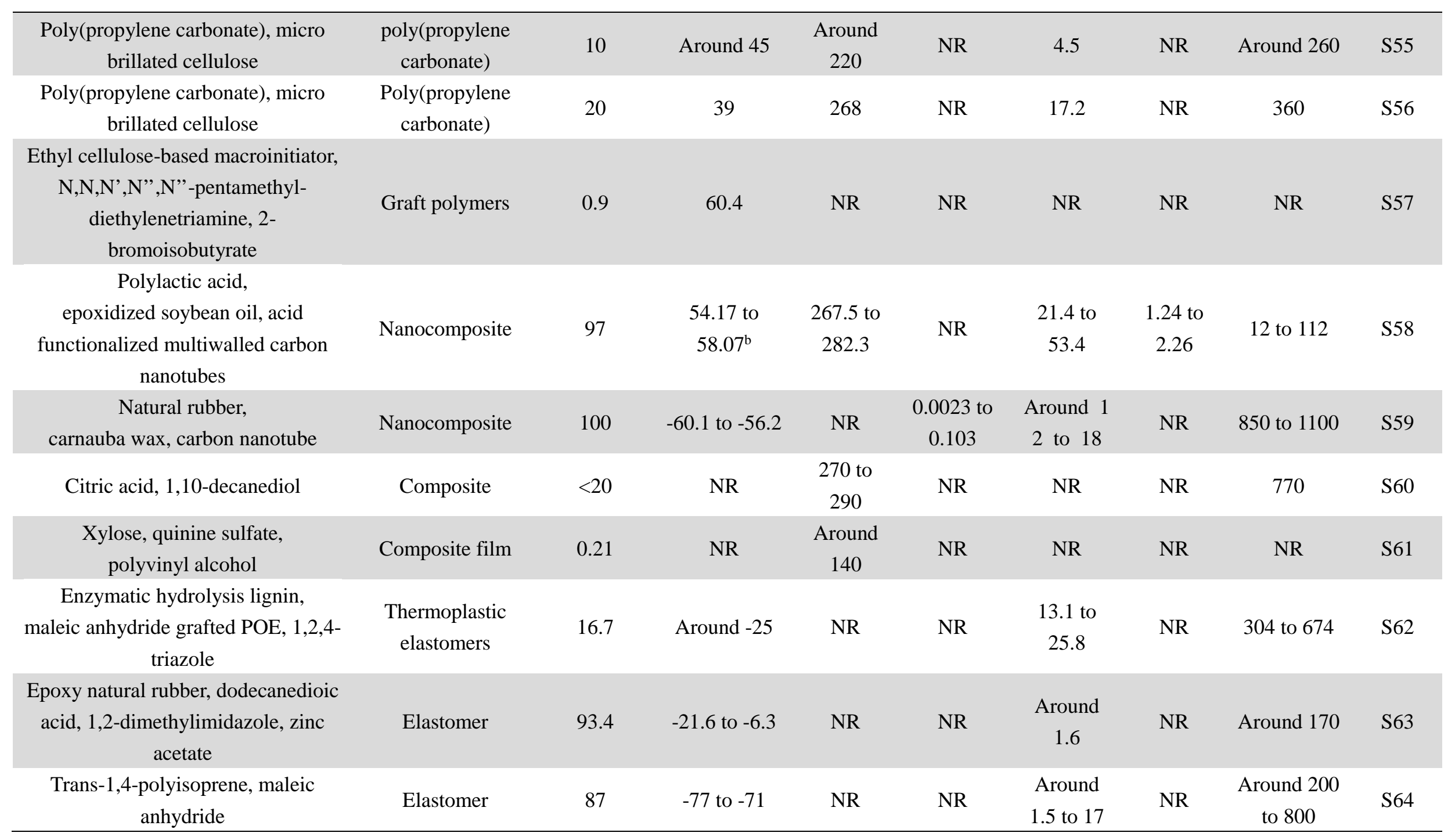




\begin{tabular}{|c|c|c|c|c|c|c|c|c|c|}
\hline $\begin{array}{l}\text { 1,4-Butanedio, 1,3-propanediol, } \\
\text { tetrabutyltitanate, hexamethylene } \\
\text { diisocyanate, -amino-4-hydroxy-6- } \\
\text { methylpyrimidine, hydroxyethyl } \\
\text { methacrylate, dibutyltin dilaurate, } \\
\text { itaconic acid, succinic acid, sebacic } \\
\text { acid }\end{array}$ & $\begin{array}{l}\text { Copolymer } \\
\text { elastomer }\end{array}$ & 56 & Around -20 & NR & NR & 0.9 to 5.2 & NR & 150 to 200 & S65 \\
\hline $\begin{array}{c}\text { Fatty acid precursors, styrene, } \\
\text { divinylbenzene }\end{array}$ & Acrylate resin & 62 & 75 & NR & NR & NR & NR & 20.1 & S66 \\
\hline Biobased polyester, boehmite & $\begin{array}{l}\text { Poly(propylene } \\
\text { sebacate) }\end{array}$ & 90 & $<100$ & NR & NR & $0.8-2.9$ & NR & $120-200$ & S67 \\
\hline $\begin{array}{c}\text { 1,6-Hexanediol, 2,5- } \\
\text { furandicarboxylic acid } \\
\text { poly(ethylene glycol), tetrabutyl } \\
\text { titanate }\end{array}$ & Copolymer & 99 & NR & $\begin{array}{c}353 \text { to } \\
364\end{array}$ & NR & 7 to 28 & NR & 31 to 710 & S68 \\
\hline $\begin{array}{c}\text { Ethylene glycol, 2,5- } \\
\text { furandicarboxylic acid } \\
\text { Poly(ethylene glycol), tetrabutyl } \\
\text { titanate }\end{array}$ & Copolymer & 99 & $78.5-87$ & $\begin{array}{l}345- \\
365.4\end{array}$ & NR & 8 to 27 & NR & 4 to 61 & S69 \\
\hline $\begin{array}{l}\text { Cellulose, poly(n-butyl acrylate-co- } \\
\text { 1-vinylimidazole) }\end{array}$ & $\begin{array}{c}\text { Poly(n-butyl } \\
\text { acrylate-co-1- } \\
\text { vinylimidazole) } \\
\text { copolymer }\end{array}$ & 0.7 & 25 to 80 & NR & NA & Around 20 & NR & $\begin{array}{c}\text { Around } \\
320\end{array}$ & S70 \\
\hline
\end{tabular}

(a) $T_{g}$ is assigned as the temperature at the maximum of $\tan \delta$-temperature curve from DMA tests except otherwise stated.

(b) $T_{g}$ is determined by DSC with heated at a rate of $10^{\circ} \mathrm{C} /$ min under a $\mathrm{N}_{2}$ atmosphere.

(c) $T_{g}$ is as the temperature at the maximum of loss modulus curve from DMA. 
(d) $T_{d i}$ is assigned as the temperature when the weight loss is $5 \mathrm{wt} \%$ except otherwise stated.

(e) NR = Not reported

(f) Storage modulus was tested at the room temperature except otherwise stated. 


\section{References}

[S1] Sha, X.-L.; Yuan, L.; Liang, G.; Gu, A. Preparation of high performance bio-based benzoxazine resin through a green solvent-free strategy for shape memory application. Polymer 2020, 202, 122673.

[S2] Tsujimoto, T.; Uyama, H. Full biobased polymeric material from plant oil and poly(lactic acid) with a shape memory property. ACS Sustainable Chem. Eng. 2014, 2, 2057-2062.

[S3] Wu, J.; Yu, X.; Zhang, H.; Guo, J.; Hu, J.; Li, M.-H. Fully biobased vitrimers from glycyrrhizic acid and soybean oil for self-healing, shape memory, weldable, and recyclable materials. ACS Sustainable Chem. Eng. 2020, 8, 6479-6487.

[S4] Yang, X.; Guo, L.; Xu, X.; Shang, S.; Liu, H. A fully bio-based epoxy vitrimer: Self-healing, triple-shape memory and reprocessing triggered by dynamic covalent bond exchange. Mater. Design. 2020, 186, 108248.

[S5] Xu, Y.; Odelius, K.; Hakkarainen, M. One-pot synthesis of lignin thermosets exhibiting widely tunable mechanical properties and shape memory behavior. ACS Sustainable Chem. Eng. 2019, 7, 13456-13463.

[S6] Yuan, D.; Chen, Z.; Xu, C.; Chen, K.; Chen, Y. Fully biobased shape memory material based on novel cocontinuous structure in poly(lactic acid)/natural rubber tpvs fabricated via peroxideinduced dynamic vulcanization and in situ interfacial compatibilization. ACS Sustainable Chem. Eng. 2015, 3, 2856-2865.

[S7] Li, H.; Sivasankarapillai, G.; McDonald, A. G. Highly biobased thermally-stimulated shape memory copolymeric elastomers derived from lignin and glycerol-adipic acid based hyperbranched prepolymer. Ind. Crop. Prod. 2015, 67, 143-154.

[S8] Zhu, Y.; Gao, F.; Zhong, J.; Shen, L.; Lin, Y. Renewable castor oil and dl-limonene derived fully bio-based vinylogous urethane vitrimers. Eur. Polym. J. 2020, 135, 109865.

[S9] Feng, X.; Fan, J.; Li, A.; Li, G. Biobased tannic acid cross-linked epoxy thermosets with hierarchical molecular structure and tunable properties: Damping, shape memory, and recyclability. ACS Sustainable Chem. Eng. 2019, 8, 874-883.

[S10] Mu, S.; Zhang, Y.; Zhou, J.; Wang, B.; Wang, Z. Recyclable and mechanically robust palm oil-derived epoxy resins with reconfigurable shape-memory properties. ACS Sustainable Chem. Eng. 2020, 8, 5296-5304.

[S11] Feng, Y.; Hu, Y.; Man, L.; Yuan, T.; Zhang, C.; Yang, Z. Biobased thiol-epoxy shape memory networks from gallic acid and vegetable oils. Eur. Polym. J. 2019, 112, 619-628.

[S12] Zhang, S.; Liu, T.; Hao, C.; Wang, L.; Han, J.; Liu, H.; Zhang, J. Preparation of a ligninbased vitrimer material and its potential use for recoverable adhesives. Green Chem. 2018, 20, 2995-3000.

[S13] Song, F.; Li, Z.; Jia, P.; Zhang, M.; $\quad$ Bo, C.; Feng, G.; Hu, L.; Zhou, Y. Tunable "soft and stiff”, self-healing, recyclable, thermadapt shape memory biomass polymers based on multiple hydrogen bonds and dynamic imine bonds. J. Mater. Chem. A 2019, 7, 13400-13410.

[S14] Li, T.; Liu, X.; Jiang, Y.; Ma, S.; Zhu, J. Bio-based shape memory epoxy resin synthesized from rosin acid. Iran. Polym. J. 2016, 25, 957-965.

[S15] Liu, T.; Hao, C.; Wang, L.; Li, Y.; Liu, W.; Xin, J.; Zhang, J. Eugenol-derived biobased epoxy: Shape memory, repairing, and recyclability. Macromolecules 2017, 50, 8588-8597.

[S16] Kasemsiri, P.; Lorwanishpaisarn, N.; Pongsa, U.; Ando, S. Reconfigurable shape memory and 
self-welding properties of epoxy phenolic novolac/cashew nut shell liquid composites reinforced with carbon nanotubes. Polymers (Basel) 2018, 10, 482, 1-16.

[S17] Amornkitbamrung, L.; Srisaard, S.; Jubsilp, C.; Bielawski, C. W.; Um, S. H.; Rimdusit, S. Near-infrared light responsive shape memory polymers from bio-based benzoxazine/epoxy copolymers produced without using photothermal filler. Polymer 2020. 209, 122986.

[S18] Lorwanishpaisarn, N.; Kasemsiri, P.; Jetsrisuparb, K.; Knijnenburg, J. T. N.; Hiziroglu, S.; Pongsa, U.; Chindaprasirt, P.; Uyama, H. Dual-responsive shape memory and self-healing ability of a novel copolymer from epoxy/cashew nut shell liquid and polycaprolactone. Polym. Test. 2020, 81, 106159.

[S19] Huang, J.; Cao, L.; Yuan, D.; Chen, Y. Design of multi-stimuli-responsive shape memory biobased PLA/ENR/ $/ \mathrm{Fe}_{3} \mathrm{O}_{4}$ TPVs with balanced stiffness-toughness based on selective distribution of $\mathrm{Fe}_{3} \mathrm{O}_{4}$. ACS Sustainable Chem. Eng. 2018, 7, 2304-2315.

[S20] Huang, J.; Fan, J.; Yin, S.; Chen, Y. Design of remotely, locally triggered shape-memory materials based on bicontinuous polylactide/epoxidized natural rubber thermoplastic vulcanizates via regulating the distribution of ferroferric oxide. Compos. Sci. Technol. 2019, 182, 107732.

[S21] Kang, H.; Gong, M.; Xu, M.; Wang, H.; Li, Y.; Fang, Q.; Zhang, L. Fabricated biobased eucommia ulmoides gum/polyolefin elastomer thermoplastic vulcanizates into a shape memory material. Ind. Eng. Chem. Res. 2019, 58, 6375-6384.

[S22] Xu, P.; Ma, P.; Cai, X.; Song, S.; Zhang, Y.; Dong, W.; Chen, M. Selectively cross-linked poly(lactide)/ethylene-glycidyl methacrylate-vinyl acetate thermoplastic elastomers with partial dual-continuous network-like structures and shape memory performances. Eur. Polym. J. 2016, 84, $1-12$.

[S23] Wang, Y.; Liu, J.; Xia, L.; Shen, M.; Xin, Z. Super-tough poly(lactic acid) thermoplastic vulcanizates with heat triggered shape memory behaviors based on modified natural eucommia ulmoides gum. Polym. Test. 2019, 80, 106077.

[S24] Zhang, H.-C.; Huang, J.; Zhao, P.-F.; Lu, X. Bio-based ethylene-co-vinyl acetate/poly(lactic acid) thermoplastic vulcanizates with enhanced mechanical strength and shape memory behavior. Polym. Test. 2020, 87, 106537.

[S25] Chen, Y.; Chen, K.; Wang, Y.; Xu, C. Biobased heat-triggered shape-memory polymers based on polylactide/epoxidized natural rubber blend system fabricated via peroxide-induced dynamic vulcanization: Co-continuous phase structure, shape memory behavior, and interfacial compatibilization. Ind. Eng. Chem. Res. 2015, 54, 8723-8731.

[S26] Magliozzi, F.; Scali, A.; Chollet, G.; Montarnal, D.; Grau, E.; Cramail, H. Hydrolyzable biobased polyhydroxyurethane networks with shape memory behavior at body temperature. ACS Sustainable Chem. Eng. 2020, 8, 9125-9135.

[S27] Dou, H.-M.; Ding, J.-H.; Chen, H.; Wang, Z.; Zhang, A. F.; Yu, H.-B. Bio-based, biodegradable and amorphous polyurethanes with shape memory behavior at body temperature. RSC Adv. 2019, 9, 13104-13111.

[S28] Cui, B.; Wu, Q.-Y.; Gu, L.; Shen, L.; Yu, H.-b. High performance bio-based polyurethane elastomers: Effect of different soft and hard segments. Chinese. J. Polym. Sci. 2016, 34, 901-909.

[S29] Kalita, H.; Karak, N. Bio-based elastomeric hyperbranched polyurethanes for shape memory application. Iran. Polym. J. 2012, 21, 263-271.

[S30] Stadler, B. M.; Brandt, A.; Kux, A.; Beck, H.; de Vries, J. G. Properties of novel polyesters made from renewable 1,4-pentanediol. ChemSusChem 2020, 13, 556-563. 
[S31] Kawaguchi, K.; Iijima, M.; Miyakawa, H.; Ohta, M.; Muguruma, T.; Endo, K.; Nakazawa, F.; Mizoguchi, I. Effects of chitosan fiber addition on the properties of polyurethane with thermo-responsive shape memory. J. Biomed. Mater. Res. B 2017, 105, 1151-1156.

[S32] Calvo-Correas, T.; Garrido, P.; Alonso-Varona, A.; Palomares, T.; Corcuera, M. A.; Eceiza, A. Biocompatible thermoresponsive polyurethane bionanocomposites with chitin nanocrystals. $J$. Appl. Polym. Sci. 2019, 136, 47430.

[S33] Kalita, H.; Karak, N. Biobased hyperbranched shape-memory polyurethanes: Effect of different vegetable oils. J. Appl. Polym. Sci. 2014, 131, 39579

[S34] Choi, K. K.; Park, S. H.; Oh, K. W.; Kim, S. H. Effect of castor oil/polycaprolactone hybrid polyols on the properties of biopolyurethane. Macromol. res. 2015, 23, 333-340.

[S35] Zhu, Y.; Gao, F.; Wei, X.; Cheng, Q.; Zhao, J.; Cao, Z.; Cao, J.; Zhong, J.; Lin, C.; Shu, J.; Shen, L. A novel bio-based coating material prepared from modified acetoacetylated castor oil and diisocyanate. Prog. Org. Coat. 2020, 138, 105397.

[S36] Petrovic, Z. S.; Milic, J.; Zhang, F.; Ilavsky, J. Fast-responding bio-based shape memory thermoplastic polyurethanes. Polymer (Guildf) 2017, 121, 26-37.

[S37] Zhang, L.; Huang, M.; Yu, R.; Huang, J.; Dong, X.; Zhang, R.; Zhu, J. Bio-based shape memory polyurethanes (bio-SMPUs) with short side chains in the soft segment. J. Mater. Chem. A 2014, 2, 11490-11498.

[S38] Gu, L.; Cui, B.; Wu, Q.-Y.; Yu, H. Bio-based polyurethanes with shape memory behavior at body temperature: Effect of different chain extenders. RSC Adv. 2016, 6, 17888-17895.

[S39] Ghosh, T.; Karak, N. Silicone-containing biodegradable smart elastomeric thermoplastic hyperbranched polyurethane. ACS Omega 2018, 3, 6849-6859.

[S40] Pattamaprom, C.; Wu, C. H.; Chen, P. H.; Huang, Y. L.; Ranganathan, P.; Rwei, S. P.; Chuan, F. S. Solvent-free one-shot synthesis of thermoplastic polyurethane based on bio-poly(1,3propylene succinate) glycol with temperature-sensitive shape memory behavior. ACS Omega 2020 , 5, 4058-4066.

[S41] Zhang, C.; Madbouly, S. A.; Kessler, M. R. Biobased polyurethanes prepared from different vegetable oils. ACS Appl. Mater. Interfaces 2015, 7, 1226-1233.

[S42] Bi, H.; Xu, M.; Ye, G.; Guo, R.; Cai, L.; Ren, Z. Mechanical, thermal, and shape memory properties of three-dimensional printing biomass composites. Polymers (Basel) 2018, 10, 1234, 114.

[S43] Merlettini, A.; Gigli, M.; Ramella, M.; Gualandi, C.; Soccio, M.; Boccafoschi, F.; Munari, A.; Lotti, N.; Focarete, M. L. Thermal annealing to modulate the shape memory behavior of a biobased and biocompatible triblock copolymer scaffold in the human body temperature range. Biomacromolecules 2017, 18, 2499-2508.

[S44] Ghosh, T.; Karak, N. Tough interpenetrating polymer network of silicone containing polyurethane and polystyrene with self-healing, shape memory and self-cleaning attributes. RSC Adv. 2018, 8, 17044-17055.

[S45] Lai, S.-M.; Wu, W.-L.; Wang, Y.-J. Annealing effect on the shape memory properties of polylactic acid (PLA)/thermoplastic polyurethane (TPU) bio-based blends. J. Polym. Res. 2016, 23, 99, 1-13.

[S46] Khasraghi, S. S.; Shojaei, A.; Sundararaj, U. Bio-based UV curable polyurethane acrylate: Morphology and shape memory behaviors. Eur. Polym. J. 2019, 118, 514-527.

[S47] Shi, S.; Wu, Q.-Y.; Gu, L.; Zhang, K.; Yu, H. Bio-based (co)polylactide-urethane networks with shape memory behavior at body temperature. RSC Adv. 2016, 6, 79268-79274. 
[S48] Hu, X.; Kang, H.; Li, Y.; Li, M.; Wang, R.; Xu, R.; Qiao, H.; Zhang, L. Direct copolycondensation of biobased elastomers based on lactic acid with tunable and versatile properties. Polym. Chem. 2015, 6, 8112-8123.

[S49] Guo, W.; Shen, Z.; Guo, B.; Zhang, L.; Jia, D. Synthesis of bio-based copolyester and its reinforcement with zinc diacrylate for shape memory application. Polymer 2014, 55, 4324-4331.

[S50] Kang, H.; Li, M.; Tang, Z.; Xue, J.; Hu, X.; Zhang, L.; Guo, B. Synthesis and characterization of biobased isosorbide-containing copolyesters as shape memory polymers for biomedical applications. J. Mater. Chem. B 2014, 2, 7877-7886.

[S51] Guo, B.; Chen, Y.; Lei, Y.; Zhang, L.; Zhou, W. Y.; Rabie, A. B.; Zhao, J. Biobased poly(propylene sebacate) as shape memory polymer with tunable switching temperature for potential biomedical applications. Biomacromolecules 2011, 12, 1312-1321.

[S52] [Tang, Z.; Kang, H.; Wei, Q.; Guo, B.; Zhang, L.; Jia, D. Incorporation of graphene into polyester/carbon nanofibers composites for better multi-stimuli responsive shape memory performances. Carbon 2013, 64, 487-498.

[S53] Zhang, Z.-X.; Dou, J.-X.; He, J.-H.; Xiao, C.-X.; Shen, L.-Y.; Yang, J.-H.; Wang, Y.; Zhou, Z.-W. Electrically/infrared actuated shape memory composites based on a bio-based polyester blend and graphene nanoplatelets and their excellent self-driven ability. J. Mater. Chem. C 2017, 5, 4145-4158.

[S54] Liu, H.; Mohsin, N.; Kim, S.; Chung, H. Lignin, a biomass crosslinker, in a shape memory polycaprolactone network. J. Polym. Sci. Part A: Polym. Chem. 2019, 57, 2121-2130.

[S55] Qi, X.; Jing, M.; Liu, Z.; Dong, P.; Liu, T.; Fu, Q. Microfibrillated cellulose reinforced biobased poly(propylene carbonate) with dual-responsive shape memory properties. RSC Adv. 2016, 6, 7560-7567.

[S56] Qi, X.; Yang, G.; Jing, M.; Fu, Q.; Chiu, F.-C. Microfibrillated cellulose-reinforced bio-based poly(propylene carbonate) with dual shape memory and self-healing properties. J. Mater. Chem. A 2014, 2, 20393-20401.

[S57] Lu, C.; Liu, Y.; Yu, J.; Wang, C.; Wang, J.; Chu, F. Fabrication of well-defined shape memory graft polymers derived from biomass: An insight into the effect of side chain architecture on shape memory properties. J. Polym. Sci. Part A: Polym. Chem. 2018, 56, 1711-1720.

[S58] Raghunath, S.; Kumar, S.; Samal, S. K.; Mohanty, S.; Nayak, S. K. PLA/ESO/MWCNT nanocomposite: A study on mechanical, thermal and electroactive shape memory properties. $J$. Polym. Res. 2018, 25, 126, 1-12.

[S59] Lai, S.-M.; Guo, G.-L.; Han, K.-T.; Huang, P.-S.; Huang, Z.-L.; Jiang, M.-J.; Zou, Y.-R. Properties and characterization of near infrared-triggered natural rubber (NR)/carnauba wax (CW)/carbon nanotube (CNT) shape memory bio-nanocomposites. J. Polym. Res. 2019, 26, 283, 116.

[S60] Fan, G.; Yang, H.; Diao, Y.; Tian, S.; Yang, T.; Sun, Q.; Liu, C.; Liu, X.; Wang, Y.; Shao, C.; Liu, C.; Liu, H.; Liu, W.; Huang, M.; Liu, H.; Chen, J.; Song, Y. Inhibitednanophase-separation modulated polymerization for recoverable ultrahigh-strain biobased shape memory polymers. Mater. Horiz. 2020,10, 2760-2767.

[S61] Zhu, Z.; Yang, P.; Chen, M.; Zhang, T.; Cao, Y.; Zhang, W.; Zhou, X.; Chen, W. Microwave synthesis of amphiphilic carbon dots from xylose and construction of luminescent composites with shape recovery performance. J. Lumin. 2019, 213, 474-481.

[S62] Huang, J.; Liu, W.; Qiu, X. High performance thermoplastic elastomers with biomass lignin as 
plastic phase. ACS Sustainable Chem. Eng. 2019, 7, 6550-6560.

[S63] Feng, Z.; Hu, J.; Zuo, H.; Ning, N.; Zhang, L.; Yu, B.; Tian, M. Photothermal-induced self-healable and reconfigurable shape memory bio-based elastomer with recyclable ability. ACS Appl. Mater. Interfaces 2019, 11, 1469-1479.

[S64] Tsujimoto, T.; Toshimitsu, K.; Uyama, H.; Takeno, S.; Nakazawa, Y. Maleated trans -1,4polyisoprene from eucommia ulmoides oliver with dynamic network structure and its shape memory property. Polymer 2014, 55, 6488-6493.

[S65] Liu, B.; Tang, Z.; Wang, Z.; Zhang, L.; Guo, B. Integrating transient and sacrificial bonds into biobased elastomers toward mechanical property enhancement and macroscopically responsive property. Polymer 2019, 184, 121914.

[S66] Capiel, G.; Marcovich, N. E.; Mosiewicki, M. A. Shape memory polymer networks based on methacrylated fatty acids. Eur. Polym. J. 2019, 116, 321-329.

[S67] Guo, W.; Kang, H.; Chen, Y.; Guo, B.; Zhang, L. Stronger and faster degradable biobased poly(propylene sebacate) as shape memory polymer by incorporating boehmite nanoplatelets. ACS Appl. Mater. Interfaces 2012, 4, 4006-4014.

[S68] Wang, G.; Jiang, M.; Zhang, Q.; Wang, R.; Qu, X.; Zhou, G. Biobased multiblock copolymers: Synthesis, properties and shape memory behavior of poly(hexamethylene 2,5furandicarboxylate)-b-poly(ethylene glycol). Polym. Degrad. Stabil. 2018, 153, 292-297.

[S69] Wang, G.; Jiang, M.; Zhang, Q.; Wang, R.; Zhou, G. Biobased multiblock copolymers: Synthesis, properties and shape memory performance of poly(ethylene 2,5-furandicarboxylate)-bpoly(ethylene glycol). Polym. Degrad. Stabil. 2017, 144, 121-127.

[S70] Wang, W.; Wang, F.; Zhang, C.; Wang, Z.; Tang, J.; Zeng, X.; Wan, X. Robust, reprocessable, and reconfigurable cellulose-based multiple shape memory polymer enabled by dynamic metal-ligand bonds. ACS Appl. Mater. Interfaces 2020, 12, 25233-25242. 